\title{
Experimental and numerical studies of process variabilities in biaxial carbon fiber braids
}

\author{
Ruben Czichos ${ }^{1} \cdot$ Oscar Bareiro ${ }^{2} \cdot$ Anthony K. Pickett $^{1} \cdot$ Peter Middendorf $^{1} \cdot$ Thomas Gries $^{2}$
}

Received: 8 August 2019 / Accepted: 17 January 2020 / Published online: 30 January 2020

(C) The Author(s) 2020

\begin{abstract}
This paper investigates the manufacture of biaxial carbon fiber braids and the influence that different machine settings have on variability of the textile architecture produced. In parallel, numerical simulations of the braiding process with these different machine settings have been conducted. For these studies yarn tension and process speed are varied to generate cylindrical biaxial braids with an average braid angle of $\pm 45^{\circ}$. The overall preform quality is characterized by means of variability in braid angle, yarn width, cover factor and fiber damage, using a variety of experimental techniques. Furthermore, from the final infused composite variations in yarn cross-section dimensions have been measured. A method is presented to transfer braid process simulation results to a detailed three dimensional finite element model of the architecture using a technique based on thermal expansion and compaction simulation. This method also allows the possibility to introduce experimentally observed variability in yarn cross-section dimensions. Such a model provides a valuable starting point for mesoscopic infusion or mechanical analysis of the textile composite. A comparison between experimental and numerical results shows that the process simulation can well reproduce the real braid angles in terms of mean value and scatter under different machine configurations and that the meso-scale textile model gives a good reproduction of the true textile architecture.
\end{abstract}

Keywords Braiding $\cdot$ Process simulation $\cdot$ Yarn architecture $\cdot$ Variability $\cdot$ Imperfection $\cdot$ Textile composites

\section{Introduction}

Braiding is a traditional hand craft used to produce ropes dating back to prehistoric times. During the past two centuries braiding machines have been developed that have continuously matured into modern highly automated systems. One important application of these machines is found in the composites industry to produce biaxial and triaxial braids by mixing stationary yarns for axial $\left(0^{\circ}\right)$ reinforcement with off-axis yarns created by the movement of radial bobbins. Today, braiding is an important manufacturing option for textile preforms that offers numerous advantages over conventional draped fabrics: Near net shaped preforms can be produced, that can be open or have a closed form section with variable

Ruben Czichos

czichos@ifb.uni-stuttgart.de

1 Institute of Aircraft Design (IFB), University of Stuttgart, Pfaffenwaldring 31, 70569 Stuttgart, Germany

2 Institut für Textiltechnik (ITA), RWTH Aachen University, Otto-Blumenthal-Straße 1, 52074 Aachen, Germany shape and diameter along its length. Over-braiding is possible to build-up reinforcement layers, and considerable flexibility in yarn architecture is achieved by control of the ratio of active axial to off-axis yarns, and control of the relative speed of circumferential bobbins to the axial take-up velocity. Mechanical properties of braided composites can be competitive with other textile composites. Indeed, the high degree of yarn interlacing does improve certain properties such as delamination toughness, damage tolerance and energy absorption for impact and crash applications. In short, braiding is an efficient, cost effective means to manufacture open or sectional preforms for advanced applications in engineering, sports equipment, marine, automotive, aerospace and other sectors.

The design of a braided structure is usually a 'trial and error' process involving trial manufacture, resin infusion and mechanical testing to determine stiffnesses and failure limit loads. Alternatively, numerical methods can be applied which, today, is feasible by undertaking three main steps as illustrated in Fig. 1. The first step involves the braiding process itself, where numerical prediction of the yarn architecture is produced [1]. If a mechanical analysis of this architecture is required it is first necessary to extract and compact the 


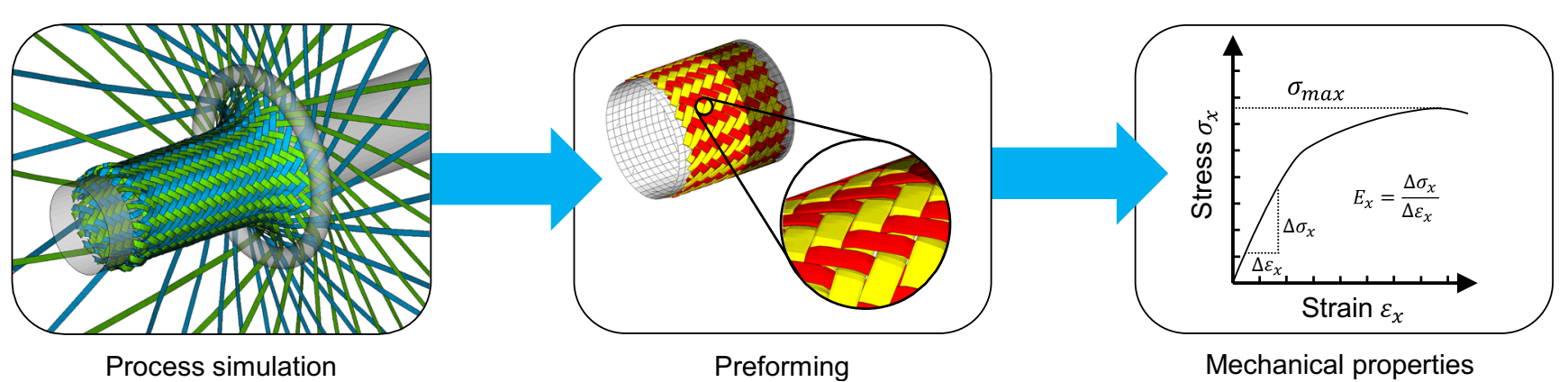

Fig. 1 Virtual braiding process chain from braid architecture to mechanical properties

architecture so it approximates its compressed state in RTM infusion tooling prior to infusion, so that it represents the architecture in the final composite. Next a finite element mesh of the superimposed matrix must be added. This can be challenging and will often lead to poor element quality and unrealistic results in damage modelling [2]; consequently, other investigators have used superposition to overlay a stiffness representation of the cured resin $[2,3]$. Finally, with appropriate constitutive models this idealisation of the composite can be used to estimate mechanical properties like stiffness and failure. The first two steps of this process chain can result in a large number of process induced variations, which can have a considerable influence on the mechanical properties $[4,5]$. The present paper therefore focuses on a representation of braids that includes manufacturing defects that can be applied at the component level.

\section{General information and process variabilities}

Braid manufacture involves opposing sinusoidal rotation of bobbins that cause yarns to slide over each other in opposing directions and to interlace, possibly combined with the interlacing of standing yarns. These motions cause frictional sliding that does result in surface fiber damage. Internal yarn fiber damage will also occur due to bending as yarns move over the bobbin rings and interlace over the braiding ring and on to the mandrel. Other defects mostly involve variability of yarn dimensions, variability of braid angles and irregular coverage leaving a poor distribution of inter-yarn gaps. The main causes for these defects are roving tension, unwinding angle, friction, take-off speed and braiding ring position [6].

Heieck [4] developed a measurement system to capture and analyze manufacturing variabilities in the final preform architecture for braids. The yarn tension, type of braid, braid ring diameter and braid angle were all varied and the visual preform characteristics, such as braiding angle, cover factor and undulation were investigated with regard to their influence on mechanical properties. The ratio of braiding ring to core diameter was identified as the strongest factor influencing preform quality. The specimen-specific fiber angle information enables a significantly better prognosis quality with regard to the braid strength. Heieck also investigated the influence of the cover factor on in-plane mechanical properties of biaxial and triaxial braids [5]. It was also shown that mechanical properties are greatly influenced by the type of braid and the testing direction, with biaxial braids showing a moderate variation and triaxial braids shown a much stronger variation. In both references $[4,5]$ the influence of process conditions on fiber damage was not taken into account.

Ebel [7] performed some first investigations to study the effect that different braiding yarn tensions has on process induced fiber damage. This research mainly concentrated on yarn tension and the number of contact points, without considering other influencing factors like take-up speed and braiding ring position. For a qualitative measurement of fiber damage a water absorption test was used [7], but it was noted that this test is approximate and has a high standard deviation, caused by the necessity to have ten probes to obtain a meaningful quantitative measure of broken fibers within a yarn. Matveev [8] investigated the variability of mechanical properties of woven textile composites. His work focused on the variability of single fiber strength, fiber orientation and layer shifting. The variability of the single fiber strength leads only to a very small variability of the final composite strength. Variations in fiber angle only showed a small influence on the composite stiffness but, similar to [4], a larger influence on the composite strength was observed. The layer shifting was found to cause a change in the shape of the stress-strain curve. The influence of the process parameters was not examined in this work. From $\mu \mathrm{CT}$ scans, Matveev [8] also noted that no variation was measurable at unit cell level. He therefore suggests that it is necessary to investigate at a large dimension scale in order to detect variations in the preform architecture.

\section{Numerical process simulations}

In recent years considerable interest has focussed on numerical analysis of the braiding process to predict yarn architecture and to detect possible imperfections such as poor compaction, bridging at sharp changes in geometry, insufficient fit to the mandrel or unacceptable spacing of yarns. This numerical 
work has used either approximate kinematic methods or more sophisticated finite element (FE) based approaches.

In the literature numerous publications provide analytical formulae to estimate yarn paths [9-12]. Some attempts have been made to improve accuracy of these analytical methods by incorporating process effects; for example van Ravenhorst et al. [13] provides an analytical solution that approximates friction effects between the yarns. However, all these methods are based on kinematic assumptions and can therefore only approximate yarn geometry since mechanical properties of yarns, their interaction during processing and proper account of friction are all ignored. A finite element simulation can overcome these limitations and several approaches are found in the literature. The most common model uses single bar or beam elements to describe the yarns $[1,3,14-16]$. However, this method does not accurately model the true yarn dimensions; consequently, other researchers have considered a multi beam approach [17], or a representation using membrane elements [18]. Since these methods cannot accurately model yarn bending stiffness, they are only conditionally suitable for a valid process simulation. Böhler [19] investigated several modelling techniques for the yarns in braiding process simulation. This work found that a shell-based approach provides the most realistic compromise, since it is able to approximate the yarn dimensions and also capture the correct bending and friction behavior of the yarn, which are essential features for an accurate simulation of the braiding process.

\section{Textile compaction behavior}

After the final preform is manufactured it needs to be prepared for resin infusion, which usually takes place within RTM tooling. This operation compacts the textile, which should be considered in the numerical process and model preparation since it also represents the final composite textile architecture. Numerous methods can be found in the literature to describe this compaction behavior. In [20] the authors assumed an elastic transversal isotropic material behavior and used a ratio of $E_{11}$ to $E_{22}$ of 100:1 to represent the low stiffness in the yarn transverse direction. In accordance with [20], Gasser et al. [21] concluded that a yarn behaves as a three-dimensional material if the elastic shear modulus $\left(G_{12}, G_{13}, G_{23}\right)$ and the Poisson's ratio $\left(\nu_{12}, \nu_{13}, \nu_{23}\right)$ are zero and if the transverse Young's modulus $\left(E_{22}, E_{33}\right)$ are very small compared to the longitudinal Young's modulus $E_{11}$. Potluri et al. [22] compared this approach with test results and observed a high sensitivity of the assumed $E_{22}$ and $E_{33}$ values; consequently, they developed an energy based approach to describe yarn deformation behavior which yielded a good agreement between test and analysis results for the compaction of a number of single layer tests, as well as for multi-layer fabrics. In [23], Green et al. used a multi-chain approach for 3D woven fabrics which idealized each yarn by 61 chains of beam elements. A comparison of the model with CT scans showed a very good agreement for compaction as well as for the flattening behavior. Another approach has been presented by Thompson et al. [24], who developed a hyper-elastic constitutive model based on a relationship between the intra yarn volume fraction and the applied compressive load. This enabled an individual calculation of the intra yarn volume fraction and also enabled the possibility to numerically capture an intra yarn volume fraction gradient. Vinot et al. [25] used an elasto-plastic yarn material behavior with an artificial low yield strength to achieve a realistic textile geometry. Both authors [24] and [25] were able to realistically model yarn deformation and were able to show a good agreement with $\mathrm{CT}$ images.

\section{Estimation of mechanical properties}

This paper focuses on process simulation and the determination of models of the braided architecture that include process induced defects. The aim of this ongoing research is to complete the virtual process chain and link process simulation to mechanical analysis. It is therefore important that the process models developed are realistic for mechanical analysis. Considerably research has investigated the mechanical behavior of textile composites; however, a review of literature in this field finds that most work is restricted to constitutive behavior at the scale of the textile Representative Volume Element (RVE). The concept of a RVE assumes the textile structure to be periodic and representative for the entire composite structure. The starting point for all simulations is a geometric description of the textile, whereby kinematic descriptions such as [26] or [27] were primarily used. The resulting models are then meshed and used in a FE simulation [28-31] to calculate stiffness and strength. The limitation of this approach is that the process induced defects are not periodic and cannot therefore be represented at the RVE scale [8]. This is evident, for example, for the distribution of defects that can be seen in Fig. 2.

Other authors, such as [32, 33], have used $\mu$-CT scans of textiles as a basis to generate numerical models; however, the resolution of these scans has also restricted these

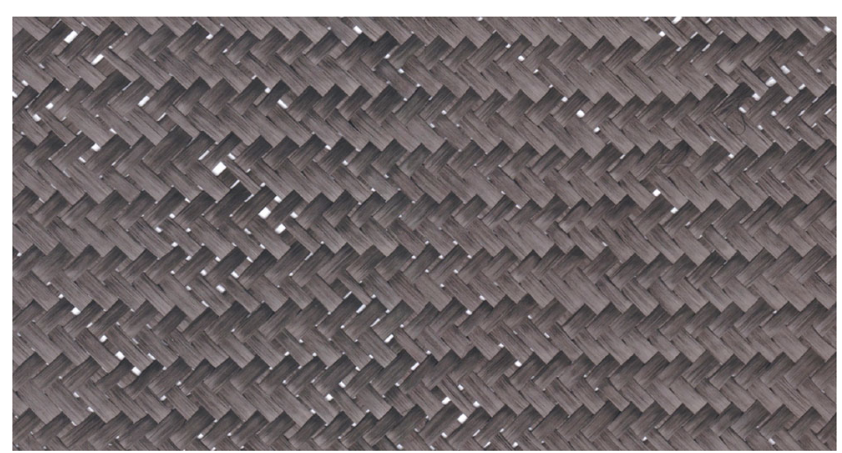

Fig. 2 Variations in a biaxial carbon fiber braid with a non-periodic distribution 
investigations to small samples and RVE elements. In the present work larger models are constructed so that the distributed effects of defects can be realistically included. Work on the coupled mechanical analysis is ongoing research that will be presented in a separate publication.

\section{Experimental investigations}

This section describes an experimental test program that has been undertaken to produce biaxial braids under different machine settings and tests that have been conducted to characterize the influence that these machine settings have on fiber damage, braid angle, yarn width and cover factor. Different methods like optical diffraction, scanning electron microscopy (SEM), computer tomography (CT) and simple optical measurements have been performed to evaluate the preform quality. After infiltration and curing, further tests have also been conducted using microsections (MS) to determine the yarn geometry of the final composite.

\section{Experimental setup and manufacturing}

Biaxial braids were manufactured at the Institut für Textiltechnik (ITA) of the RWTH Aachen University using a Herzog RF1/64-120 radial braiding machine. In accordance with [6], the braid pattern is a regular braid, which is achieved by using a fully occupied machine. For this work a simple cylindrical mandrel with a diameter of $85 \mathrm{~mm}$ was used, in order to avoid a negative effect of a complex mandrel geometry on the fiber architecture. The yarn material used is Tenax®-E HTS40 F13 $24 \mathrm{~K}$ with 1600 tex [34]. The range of machine settings investigated are shown in Table 1 . The values for yarn tension are based on the available springs at ITA, whereas the springs in the lever-balanced carriers are varied from $1.0 \mathrm{~N}$ up to $7.0 \mathrm{~N}$ in equidistant intervals. From weaving technology it is commonly known that a reduction of fiber damage for processing technical fibers such as glass or carbon, is achieved by reducing the machine speed by up to $75 \%$ compared to normal textile operation speeds [35]. From experience of the authors practical processing speeds for technical fibers are between 80 and $100 \mathrm{rpm}$. Consequently, for

Table 1 Machine configurations for the braiding process

\begin{tabular}{lll}
\hline Yarn tension $[\mathrm{N}]$ & $\begin{array}{l}\text { Horn gear rotational } \\
\text { speed [rpm] }\end{array}$ & $\begin{array}{l}\text { Mandrel take-up } \\
\text { speed }[\mathrm{mm} / \mathrm{s}]\end{array}$ \\
\hline 1.0 & 50 & 14 \\
1.0 & 150 & 40 \\
3.5 & 100 & 30 \\
7.0 & 50 & 14 \\
7.0 & 150 & 32 \\
\hline
\end{tabular}

this study $100 \mathrm{rpm}$ is taken as the mean value, with other limiting speeds of $50 \mathrm{rpm}$ and $150 \mathrm{rpm}$. A total of three individual layers per configuration were manufactured and analyzed.

In order to remove the braids from the mandrel for the preform characterization both ends were fixed on the mandrel with tape and a length of tape was applied along the longitudinal axis to prevent unwanted movements during the cutting process. In these operations great care was taken to ensure the preform was not improperly deformed during handling. Lastly, the circular preform was carefully flattened so that measurements could be taken of the textile architecture.

\section{Preform characterization}

The preforms created by the machine settings in Table 1 were analyzed. This involved a variety of techniques to assess fiber damage and an optical technique to measure variations in the architecture. In order to determine the influence of the process parameters on each individual characteristic effect diagrams were used. In general, these diagrams describe the impact that a factor has on the system, which is characterized by the socalled 'effect'. It is defined as the difference between two mean values and quantifies the average registered change of the quality characteristic when switching from the highest to the lowest machine configuration [36]. These values were then considered to identify the machine parameters that lead to the highest overall preform quality, which was defined by a global quality index.

\section{Fiber damage}

To determine the amount of fiber damage, optical diffraction, high-resolution computer tomography (CT) and scanning electron microscopy (SEM) were used.

Optical diffraction The experimental setup for optical diffraction is shown in Fig. 3. In this technique a laser emits a light beam which is broadened by a first set of lenses to create parallel light beams. The damaged yarn is mounted on a sample holder and positioned in the area of parallel light. A Fourier lens is located behind the sample at a distance $f$ to converge the parallel beams on to the focal plane. The focal plane is positioned at a distance $f$ from the Fourier lens and a sensor is placed at this distance to record the diffraction patterns generated.

The broken filaments protruding from a roving are generated as a consequence of production induced fiber damage; this is referred to as yarn hairiness and creates a distinctive diffraction pattern depending on the level of fiber damage. The damage quantification is based on an image processing algorithm that isolates and quantifies the diffraction patterns generated as a consequence of yarn hairiness. Therefore, the 

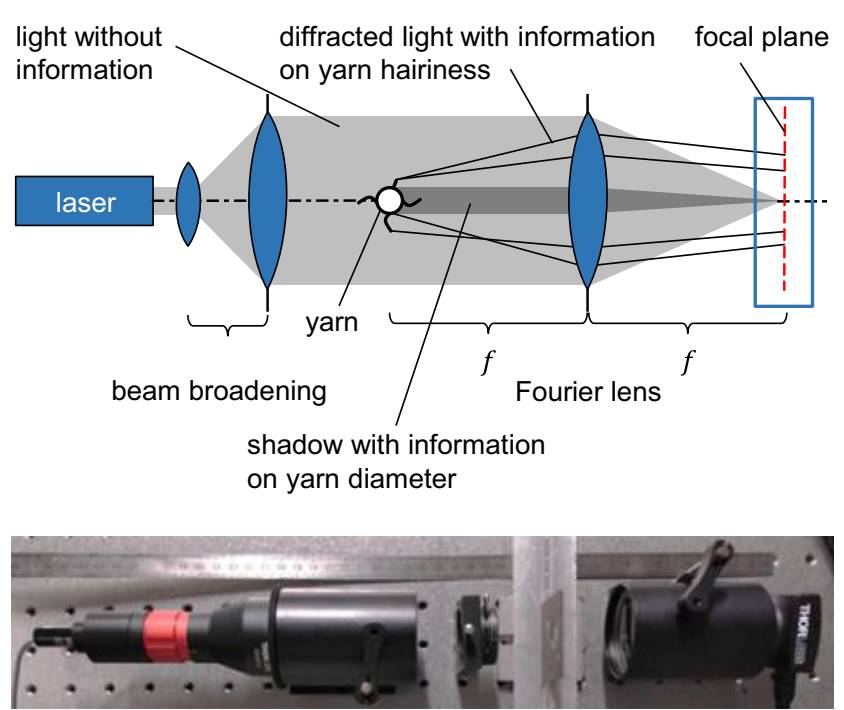

Fig. 3 Schematic representation of the measurement setup (top) and experimental setup (bottom)

captured images of the laser beam with an empty sample holder (Fig. 4a) and a yarn in pristine condition (Fig. 4b) are captured. Taking an average of 10 images, these patterns are used as a reference for analysis. By comparing Fig. 4b) and Fig. 4c) it can be observed that the presence of protruding filaments influences the diffraction pattern captured. With increase fiber breakage the greater is the diffraction points that are created, leading to a more intense dispersion on the diffraction patterns.

An image-processing algorithm written in Python generates differential images of the diffraction patterns in order to isolate the diffraction pattern that occurs as a result of yarn hairiness. In the first step the diffraction patterns from the empty sample holder and a pristine yarn are subtracted from the damaged yarn pattern, which eliminates the circular high intensity region at the center and the typical pattern of the yarn in pristine condition. Subsequently, the images are converted to normalized grey scale images with a pixel range from 0 (black pixel) to 1 (white pixel). For the fiber damage analysis, 10 different samples for each configuration were treated. One diffraction pattern was taken for each sample. The image process algorithm takes theses 10 images and produces one averaging all 10 images. The differential images are then obtained from these averaged images. The isolated diffraction patterns of the damaged yarns can be seen in Fig. 5 .

The intensity of the diffraction patterns is used to determine a fiber damage index $(F D I)$, which is defined as the mean pixel value. From Fig. 5 it can be seen that the diffraction pattern appears in lighter grey shades (closer to white) when the amount of fiber damage increases. Values for the effect of fiber damage depending on individual machine settings are listed in Table 2 and plotted in the effect diagram in Fig. 9a).

From Table 2 and Fig. 9a) it is found that the damage indices increase for different yarn tensions independent of the process speed. This can be traced back to higher interyarn friction forces when increasing the yarn tension. Also, the process speed shows an influence on the FDI. A higher process speed tends to increase the fiber damage; this is also known for weaving of technical fibers [35]. This effect is amplified by a higher yarn tension. The type of braid does not appear to have a significant effect on fiber damage.

Computer tomography Further detection of fiber damage had been investigated using high-resolution CT-scans which were kindly performed at the European Synchrotron Radiation Facility (ESRF) in Grenoble. Due to the limited amount of radiation time available only three samples could be evaluated, which were manufactured using the following machine settings:

- 50 rpm with $1.0 \mathrm{~N}$ yarn tension

- $100 \mathrm{rpm}$ with $3.5 \mathrm{~N}$ yarn tension

- $150 \mathrm{rpm}$ with $7.0 \mathrm{~N}$ yarn tension

The enormous amount of data generated by CT did not allow manual detection of broken filaments, and therefore an evaluation algorithm developed by the Fraunhofer Institut für Wirtschaftsmathematik (ITWM) was used. For the machine configuration with $150 \mathrm{rpm}$ and $7.0 \mathrm{~N}$ yarn tension, broken
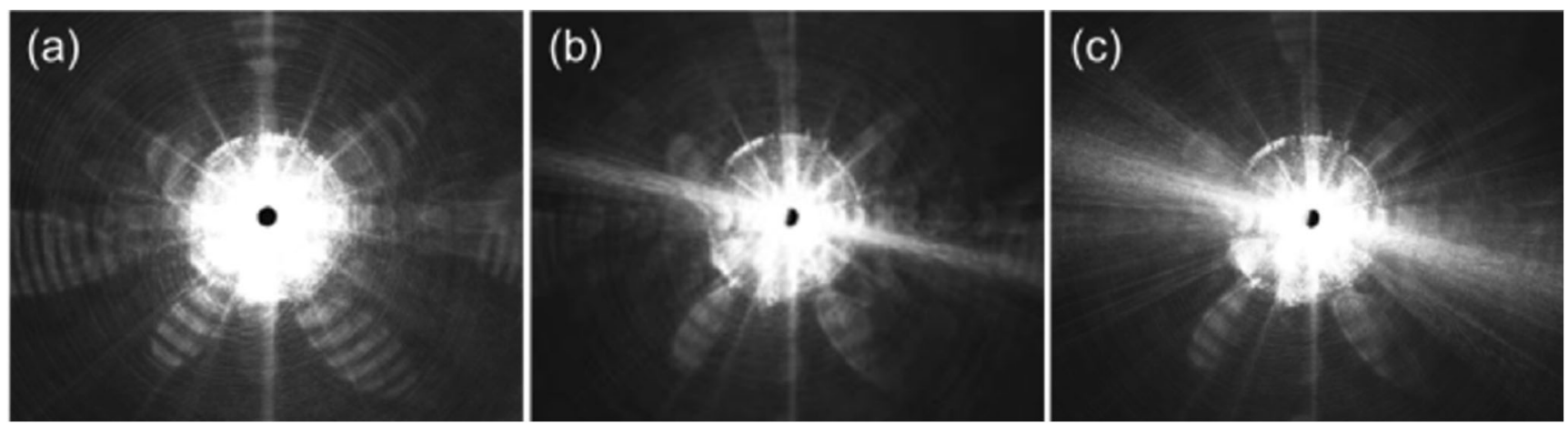

Fig. 4 Diffraction pattern: (a) from laser and empty sample holder; (b) pristine yarn; (c) damaged yarn 

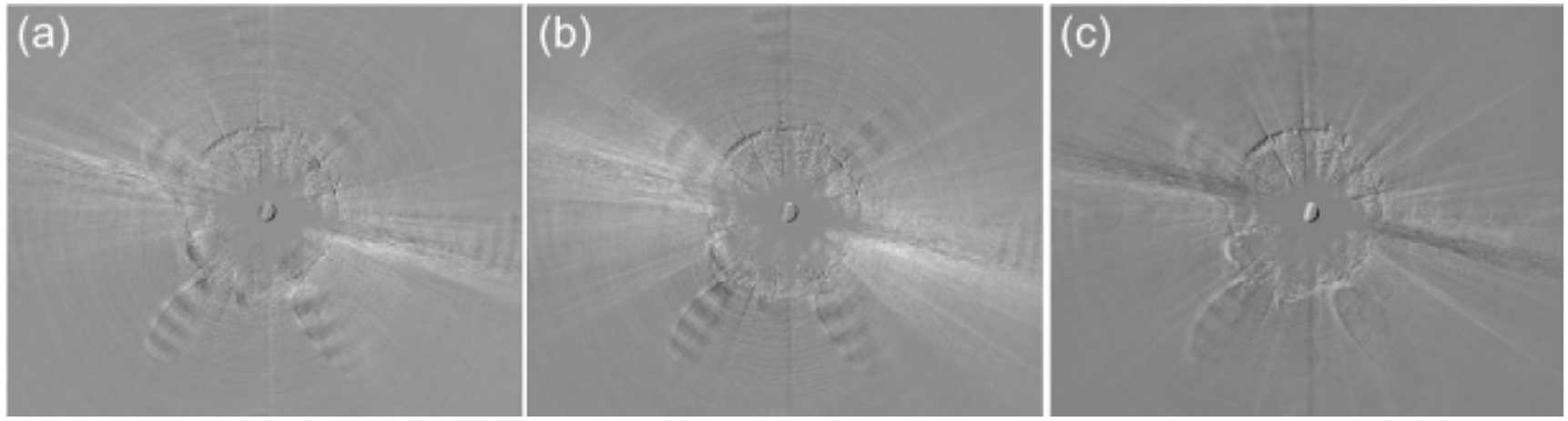

Fig. 5 Diffraction pattern of damaged yarns manufactured with: (a) $50 \mathrm{rpm} \& 1.0 \mathrm{~N}$; (b) $150 \mathrm{rpm} \& 1.0 \mathrm{~N}$; (c) $50 \mathrm{rpm} \& 7.0 \mathrm{~N}$

filaments can be detected as depicted in Fig. 6. Fiber damage appears in every area of the yarn and can be found directly on the surface between two yarns (Fig. 6a), inside the yarn but near the surface (Fig. 6b, c) and inside the yarn itself (Fig. 6d). However, since only one sample per machine setting could be examined a valid quantification of the broken filaments was not possible.

Scanning electron microscopy In addition to broken filaments damage to the filament surface sizing also occurs. This has been investigated with a scanning electron microscope (SEM). Fig. 7 displays the same yarn material under different conditions. Fig. 7a) shows the undamaged yarn, whereas Fig. 7b) indicates removal of the sizing after the rewinding step. Fig. 7c) shows a braid sample manufactured with $150 \mathrm{rpm}$ and $7.0 \mathrm{~N}$ yarn tension, where it can be observed that there is considerable damage to the sizing, which is caused by excessive friction during the braiding process. A quantification of these defects has not so far been taken into account.

\section{Yarn architecture}

For the preform characterization the samples were digitalized by scanning them with a CanoScan LiDE 200 flatbed scanner. The images were then analyzed with regard to distributions of braid angle, yarn width and cover factor. The braid angle is determined by using a tool provided by Faserinstitut Bremen e.V. (FIBRE), whose measuring principle is based on a frequency distribution of the edge orientation [37, 38]. For this work the original image is subdivided into smaller segments and the braid angle is calculated for each section, as shown in Fig. 8a). For the chosen subdivision size, each braided layer contains about 600 measuring points for the braid angle. The cover factor is evaluated by using a MATLABscript, which converts the original image into a grey-scale image containing only black and white pixels Fig. 8b). The correct allocation of the pixels is done by manually setting a threshold, which enables the pixels to be counted and assigned either to the yarns (black) or gaps (white). The ratio of the black pixels to the overall sum of pixels is defined as the cover factor. An automated measurement of the yarn width is not possible, and therefore this distribution has been determined manually using the open source software GIMP. A scale with the same resolution as that used for the preforms was scanned, which enables the conversion from the pixels into a length unit. A total of 150 measuring points per manufactured layer was captured for this textile characteristic.

The results of this preform characterization are listed in Table 2 . The present work aims to identify the optimum machine settings to produce a braid that is as homogeneous and undamaged as possible, so the chosen quality criteria for the braid angle as well as for the yarn width are the mean of the standard deviation of these two values. As a fully covered braid corresponds to the highest possible quality, the mean of the cover factor itself is chosen as a quality criterium. Most work in this study has focused on the influence of the

Table 2 Preform characterization results

\begin{tabular}{llllll}
\hline Machine Setting & Type of Braid [-] & Braid Angle [ $\left.{ }^{\circ}\right]$ & Yarn Width [mm] & Cover Factor [\%] & Fiber Damage Index [-] \\
\hline $50 \mathrm{rpm} \& 1.0 \mathrm{~N}$ & biax. & $44.87 \pm 0.64$ & $5.59 \pm 0.47$ & $99.89 \pm 0.05$ & 0.303 \\
$150 \mathrm{rpm} \& 1.0 \mathrm{~N}$ & biax. & $43.66 \pm 1.47$ & $5.45 \pm 0.56$ & $99.66 \pm 0.34$ & 0.321 \\
$100 \mathrm{rpm} \& 3.5 \mathrm{~N}$ & biax. & $42.69 \pm 0.27$ & $5.64 \pm 0.40$ & $99.72 \pm 0.09$ & 0.310 \\
$50 \mathrm{rpm} \& 7.0 \mathrm{~N}$ & biax. & $44.65 \pm 1.11$ & $5.60 \pm 0.41$ & $99.86 \pm 0.07$ & 0.318 \\
$150 \mathrm{rpm} \& 7.0 \mathrm{~N}$ & biax. & $44.76 \pm 1.48$ & $5.52 \pm 0.41$ & $99.76 \pm 0.12$ & 0.340 \\
$100 \mathrm{rpm} \& 3.5 \mathrm{~N}$ & triax. & $39.99 \pm 0.97$ & $4.72 \pm 0.40$ & $98.18 \pm 0.47$ & 0.315 \\
\hline
\end{tabular}




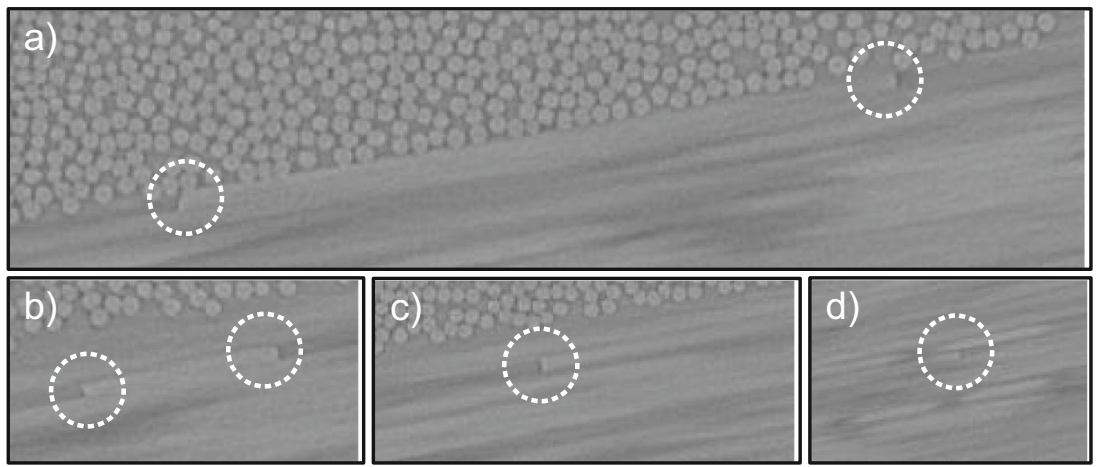

Fig. 6 Fiber damage: (a) on the surface between two yarns; (b) and (c) inside the yarn but near the surface; (d) inside the yarn

yarn tension and the process speed for biaxial braids, however, one set of triaxial braids was also manufactured to study the influence that additional standing yarns have on the braid quality.

The effect diagram for the braid angle is shown in Fig. 9b). By comparing the influence of the yarn tension it can be seen that for a low process speed a high yarn tension increases the standard deviation of the braid angle, which results in a loss of preform quality. However, by using a high process speed, no significant influence of the yarn tension on the evaluated values can be observed. In general, it can be stated that a higher process speed will increase the scatter of braid angle, which is explained by high oscillations during manufacturing causing a poor preform quality. For this limited study the comparison between different braid types shows that the standard deviation of the braid angle is much higher for a triaxial braid compared to the biaxial braids. This is attributed to the $0^{\circ}$ standing yarns in the triaxial braid, which induce greater undulations and higher friction effects, which in turn causes higher variations in the braid angle.

The effect diagram for the yarn width is displayed in Fig. 9c), where it can be seen that regardless of the process speed, a higher yarn tension always reduces the scattering of the yarn width. This is related to a higher cohesion of the single filaments within a yarn when applying a high tension. A difference between a biaxial and a triaxial braid cannot be observed.
In both cases the process parameters are kept constant and the standard deviation of the yarn width is nearly the same. It can be stated that this preform characteristic only seems to be influenced by the process itself and not by the type of braid. The effect diagram of the cover factor is shown in Fig. 9d), which shows no clear tendency by comparing different yarn tensions, however, different results can be seen when looking at the process speed. Independent of the yarn tension, a higher process speed seems to lower the cover factor. In this case the type of braid has the greatest influence on cover factor. As the triaxial braid has a higher level of undulation due to the additional set of standing yarns, the cover factor decreases as a result of an increased number of gaps.

\section{Evaluation of the preform quality}

Since all of the above mentioned factors influence preform quality, it is convenient to combine these using a single quality index as presented in [4]. Furthermore, this index is extended here by adding a factor for fiber damage. The final relation for quality index is

$Q_{t o t}=\left(2 Q\left(s_{\gamma}\right)+Q\left(s_{b}\right)+Q(c f)+Q(F D I)\right) \cdot \frac{1}{30}$

where $Q\left(s_{\gamma}\right)$ and $Q\left(s_{b}\right)$ describe the quality index of the braid angle and yarn width distribution as a function of their
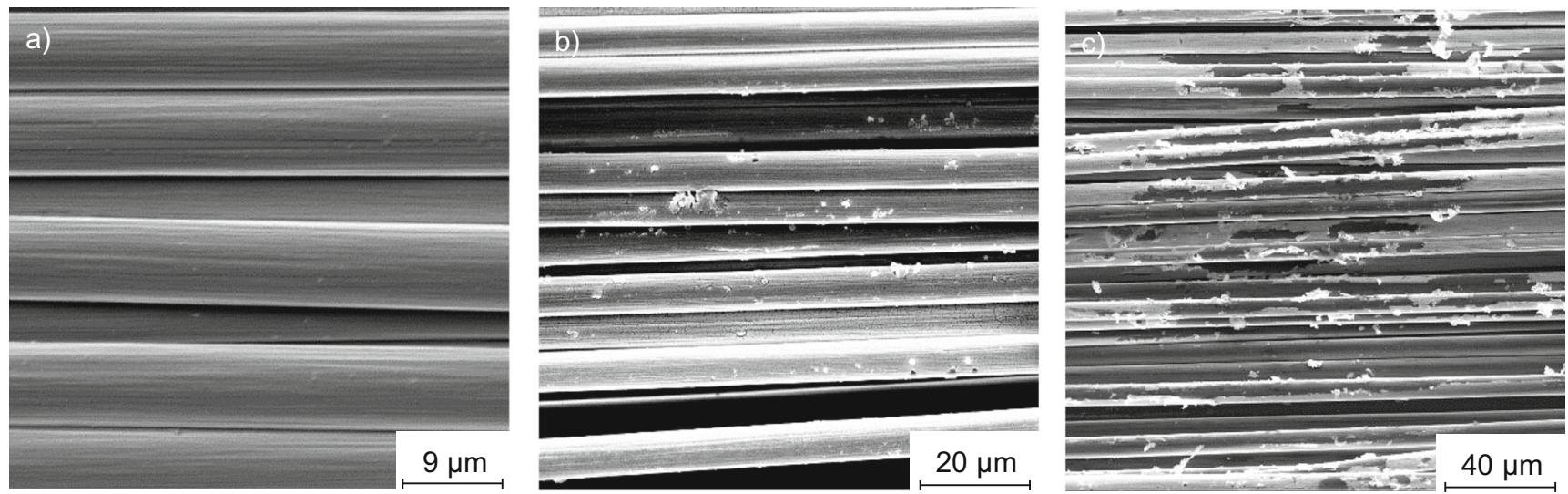

Fig. 7 (a) undamaged yarn surface; (b) microcracks on the surface after rewinding; (c) wear of the sizing after braiding 
Fig. 8 Fiber architecture analysis: (a) braid angle; (b) cover factor
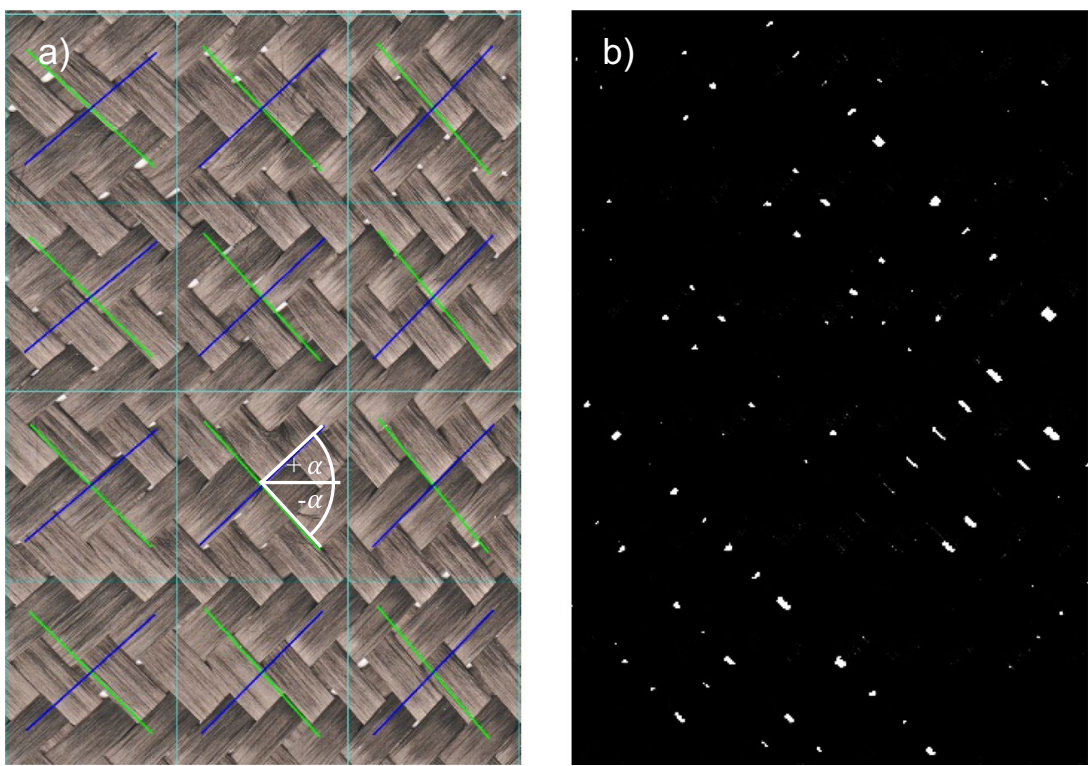

standard deviations $s_{\gamma}$ and $s_{b}$ respectively. A fully covered braid corresponds to the highest quality, so its quality index $Q(c f)$ is a function of the cover factor $c f$ itself. The quality index for the fiber damage $Q(F D I)$ is a function of the $F D I$, which is explained in "Model pre-processing and thermal expansion" section. In Eq. (1) a weighting factor of 2 is applied to the braid angle since this is considered to be the most important factor for stiffness performance, which in most cases is the basis for structural dimensioning. The individual quality indices can vary from 1 (poorest quality) to 6 (highest quality), so the overall quality index is normalized to obtain a value between 0 and 1 ,
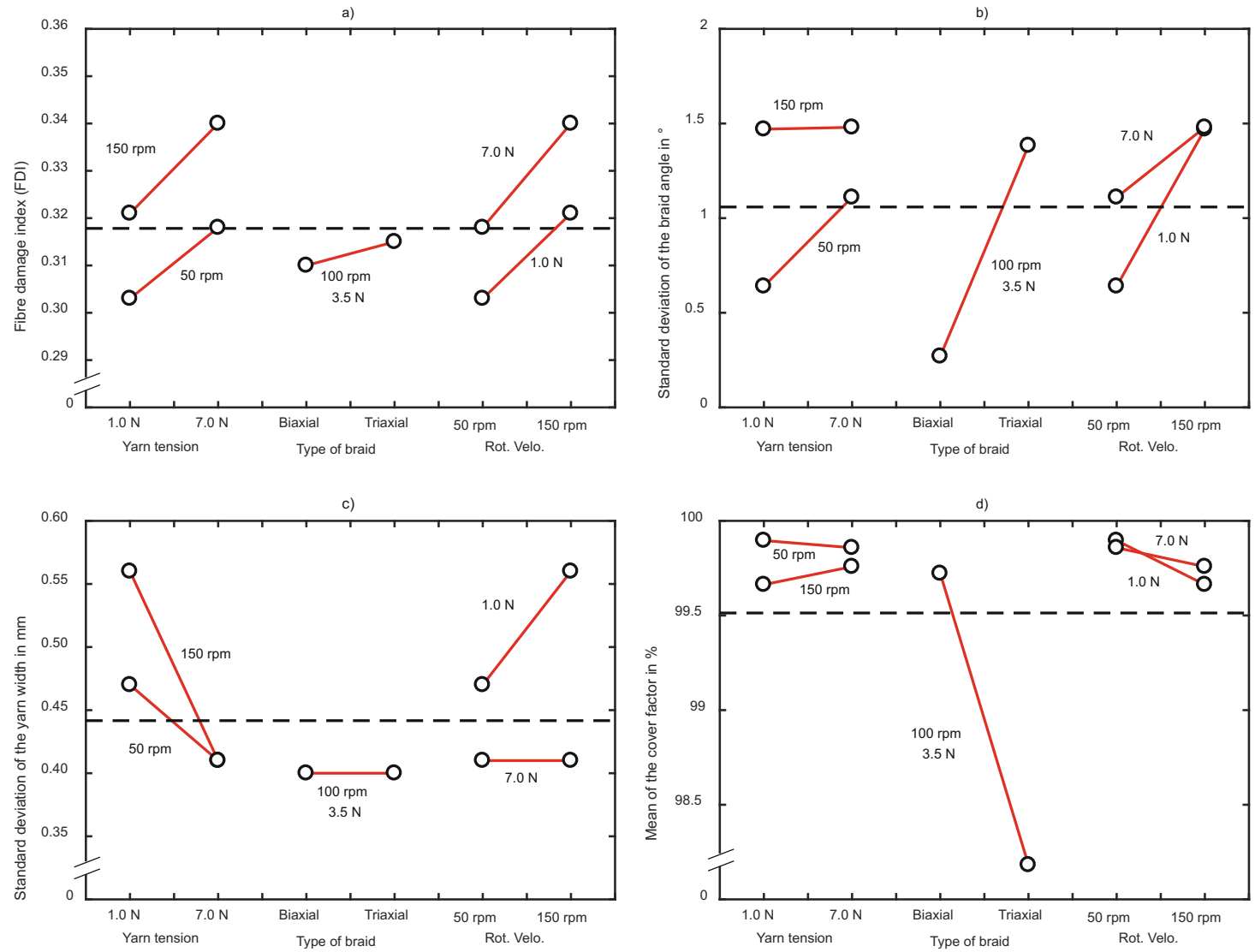

Fig. 9 Effect diagrams for: (a) fiber damage index (FDI); (b) braid angle; (c) yarn width; (d) cover factor 
Table 3 Assignment of the individual quality indices

\begin{tabular}{lllllll}
\hline $\begin{array}{l}\text { Individual } \\
\text { quality index } Q\end{array}$ & 6 & 5 & 4 & 3 & 2 & 1 \\
\hline$s_{\gamma}\left[^{\circ}\right]$ & $\leq 0.27$ & $0.28-0.57$ & $0.57-0.88$ & $0.88-1.18$ & $1.18-1.48$ & $>1.48$ \\
$s_{b}[\mathrm{~mm}]$ & $\leq 0.40$ & $0.41-0.44$ & $0.44-0.48$ & $0.48-0.52$ & $0.52-0.56$ & $>0.56$ \\
$c f[\%]$ & $\leq 99.89$ & $99.88-99.83$ & $99.83-99.78$ & $99.78-99.72$ & $99.72-99.66$ & $>99.66$ \\
$F D I[-]$ & $\leq 0.303$ & $0.304-0.312$ & $0.312-0.322$ & $0.322-0.331$ & $0.331-0.340$ & $>0.340$ \\
\hline
\end{tabular}

whereby 1 corresponds to $100 \%$ and indicates the highest preform quality possible.

The values from Table 2 are used to assign the individual quality indices by rating them from 1 to 6 , where the highest and lowest values are used for the limiting values (Table 3). Using Eq. (1) the overall preform quality is obtained, which is depicted in Fig. 10. From these results it can be concluded that higher process speeds lead to a lower preform quality. This can be traced to the high influence of the standard deviation of the braid angle, which is increased due to higher oscillations when applying a faster process speed. Using a high process speed in combination with a high yarn tension will lead to the poorest overall preform quality as additional fiber damage will occur. For the present study, the best preform quality is achieved with a medium yarn tension and a medium process speed. This seems to be contradictory, since a reduction in the preform quality would be expected when considering the respective extreme values of yarn tension and speed. However, an increase in yarn tension is also accompanied by an increase of the friction forces inside the roving, which has a positive effect on the cohesion of the single filaments during the process. A tension value of $3.5 \mathrm{~N}$ is found as an optimum for these studies in order to ensure a homogeneous braid while, at the same time, reducing fiber damage. The increased process speed causes an increase in the scattering of braid angle and yarn width, whereby the effect of lower cohesion forces at lower yarn tension is even more pronounced. This can be

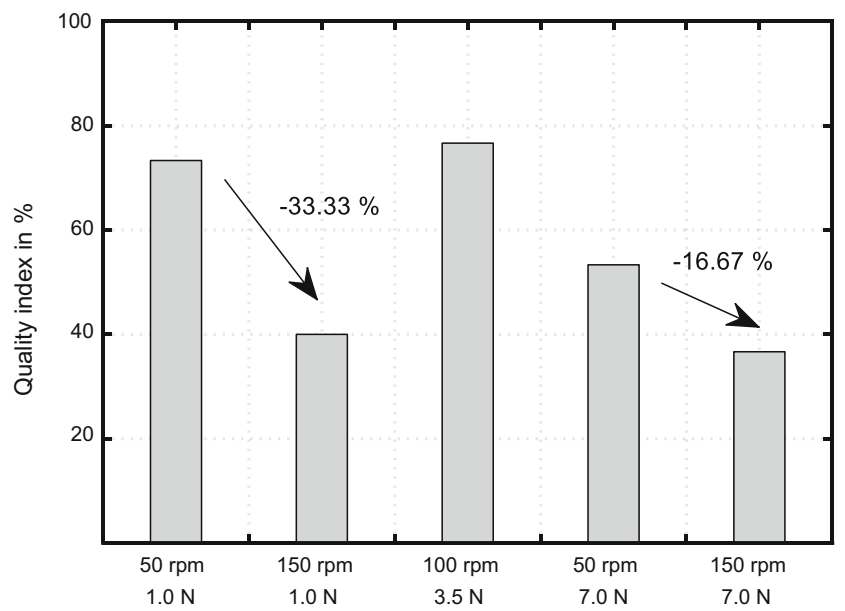

Fig. 10 Influence of process parameters on the overall preform quality indices $Q_{t o t}$ partially compensated by a higher yarn tension, whereby a too high value leads to an increase of fiber damage and thus to a decrease of the preform quality.

\section{Composite characterization}

In this section the architecture of the final textile composite is analyzed in terms of yarn width and height. These values provide important information for the numerical simulations to be presented later and are therefore of particular interest. The preforms are infiltrated with an epoxy resin EPIKOTE ${ }^{\mathrm{TM}}$ Resin MGSTM RIMR 135 with hardener EPIKURE ${ }^{\text {TM }}$ Curing Agent MGSTM RIMH 137. A Vacuum Assisted Process (VAP) is used to infuse the resin and minimize porosity. The process is done on a heated table at $30^{\circ} \mathrm{C}$ to lower resin viscosity and a glass plate is placed on the top surface to obtain a good quality finish on both sides of the samples. After degassing the resin, the final infusion pressure is set to $30 \mathrm{mbar}$. From the cured composite, microsections are prepared to measure the yarn geometry and dimensions by means of light microscopy. An example micrograph section is shown in Fig. 11. The stacking of several layers will cause nesting so that some warp/weft yarns will merge with a warp/weft yarn from the adjacent layer. These merged yarns are highlighted in red in Fig. 11 and are not used for the evaluation process.

\section{Yarn cross-section}

The distribution of yarn cross-section dimensions is analyzed only for separated yarns, which are marked in green in Fig. 11. The height and width are measured and evaluated for at least 40 yarns per machine setting. These results are listed in Table 4, with values given for the mean as well as the standard deviation of yarn dimensions plotted in Fig. 12.

By comparing the results for the different machine settings no clear tendency can be observed and the variation for both the yarn width and the yarn height are in a similar range. It can be concluded that the coefficient of variation (CV) of the yarn width is approximately $7 \%$, whereas the $\mathrm{CV}$ of the yarn height is nearly twice as high at about $14 \%$. These results match observations from [39] for a biaxial $\pm 45^{\circ}$ carbon fiber braid using a Tenax ${ }^{\circledR}$-E HTS40 F13 $12 \mathrm{~K}$ yarn with 800 tex. 
Fig. 11 Microsection with separated yarns (green) and merged yarns (red)

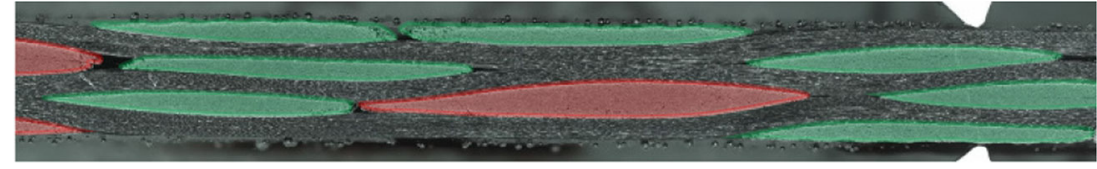

\section{Numerical investigations}

This section describes numerical simulations to obtain a realistic braided architecture that includes variabilities of braid angle and yarn cross section dimensions. After some general information on the braiding simulation, the calibration of the material model is described, followed by procedures used to generate the yarn architecture similar to the compacted form that it will have in infusion tooling and the final cured composite part.

\section{Braiding process simulation}

For braiding process simulation the explicit finite-element code LS-DYNA R10.1.0 from Livermore Software Technology Corporation (LSTC) is used. The model set-up has the same geometry and configuration as the real machine with a total of 64 yarns rotating on a sinusoidal path around the mandrel's longitudinal axis, whereby one set rotates clockwise (warp yarns) and the other set rotates counter-clockwise (weft yarns). The model creation follows similar methods to those discussed in [1], giving the final model shown in Fig. 13.

The yarns are guided through a braiding ring and are attached to the end of the mandrel, which moves with a constant axial velocity. The other end of each yarn is attached to a spring element which simulates the function of the carrier and is used to apply a yarn tension force. In accordance with [6] the main function of the carrier is to keep the yarn tension as constant as possible during the braiding process. From [6] it is known, that the yarn tension depends on the weight and the radius of the bobbin which both decrease during the unwinding process of the yarn and therefore result in a decreasing yarn tension throughout the braiding process. However, for the presented study no detailed information on the yarn tension fluctuation is available, and therefore yarn tension is assumed to be constant. This is done using a non-linear elastic spring formulation (*Mat_Spring_Nonlinear_Elastic in LSDYNA) with an assigned constant force versus displacement curve.

The braiding ring and mandrel are modelled as rigid bodies and discretized using quadrilateral shell elements. The yarns are discretized with triangular shell elements, since this element type does not suffer from hourglassing modes and provides a good compromise between accuracy and computation speed. For an accurate simulation the yarn element dimensions length $l$ and width $w$ are carefully chosen. The yarn width can be taken from its experimental value. The element length is selected to try and have a minimum number of elements in the model, without compromising the accuracy of yarns to deform to fit to curved surfaces. In this study, a maximum discretization error of $\leq 3 \%$ is demanded. Taking the smallest occurring radius of the process into account, which in this case is the braid ring radius of $r=10 \mathrm{~mm}$, the minimum required element length $l$ can be calculated by dividing the discretized area by the actual area (Eq. (2)).

$\frac{A_{\text {discretized }}}{A_{\text {actual }}}=\frac{\frac{m \cdot r^{2}}{2} \cdot \sin \left(\frac{2 \pi}{n}\right)}{\pi \cdot r^{2}} \leq 3 \%$

Equation (2) gives the minimum required number of elements $m$. By entering this value to Eq. (3) the minimum required element length $l$ for a discretization error of $\leq 3 \%$ is obtained giving $l=4.16 \mathrm{~mm}$. This value is used for meshing of the yarns.

$l=2 r \cdot \sin \left(\frac{\pi}{m}\right)$

The contact is treated with a global general contact between all parts (*Contact_Automatic_Single_Surface in LS-DYNA) with an assumed constant friction coefficient of $\mu=0.2$. As the shell elements do not have a geometrical thickness, the
Table 4 Statistical results of the yarn geometry

\begin{tabular}{|c|c|c|c|c|c|c|}
\hline \multirow[t]{2}{*}{ Machine Setting } & \multicolumn{3}{|c|}{ Yarn width } & \multicolumn{3}{|c|}{ Yarn height } \\
\hline & $\mu[\mathrm{mm}]$ & $\sigma[\mathrm{mm}]$ & $C V[\%]$ & $\mu[\mathrm{mm}]$ & $\sigma[\mathrm{mm}]$ & $C V[\%]$ \\
\hline $50 \mathrm{rpm} \& 1.0 \mathrm{~N}$ & 5.80 & 0.46 & 7.87 & 0.361 & 0.048 & 13.18 \\
\hline $150 \mathrm{rpm} \& 1.0 \mathrm{~N}$ & 5.68 & 0.44 & 7.70 & 0.377 & 0.050 & 13.16 \\
\hline $100 \mathrm{rpm} \& 3.5 \mathrm{~N}$ & 5.60 & 0.47 & 8.35 & 0.393 & 0.054 & 13.71 \\
\hline $50 \mathrm{rpm} \& 7.0 \mathrm{~N}$ & 5.74 & 0.36 & 6.23 & 0.335 & 0.051 & 15.29 \\
\hline $150 \mathrm{rpm} \& 7.0 \mathrm{~N}$ & 5.61 & 0.39 & 6.99 & 0.384 & 0.055 & 14.24 \\
\hline
\end{tabular}




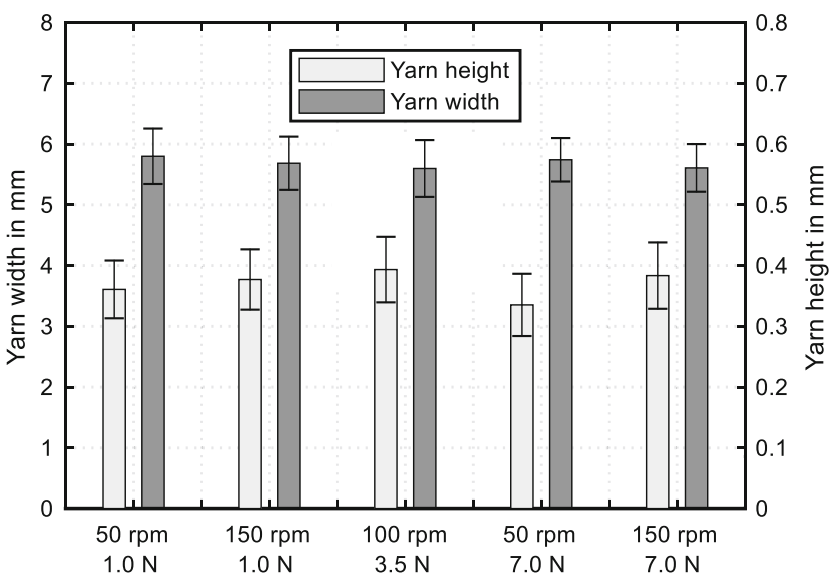

Fig. 12 Results of the yarns cross-section measurement

distance between the two interacting yarn systems is controlled by the contact thickness. This value mainly influences the undulations within the final braid and therefore must be set to a realistic value, which is equal to the physical yarn thickness. From Fig. 12 this value is set to $0.40 \mathrm{~mm}$. A small amount of viscous damping is applied in the contact definition to damp contact forces and minimize unwanted oscillations.

\section{Yarn bending behavior}

A difficulty for a realistic braiding process simulation is the correct definition of the yarns bending stiffness, since this value is usually very low in comparison to the high yarn tensile stiffness. A cantilever bending test was carried out in accordance with DIN 53362 to determine yarn bending

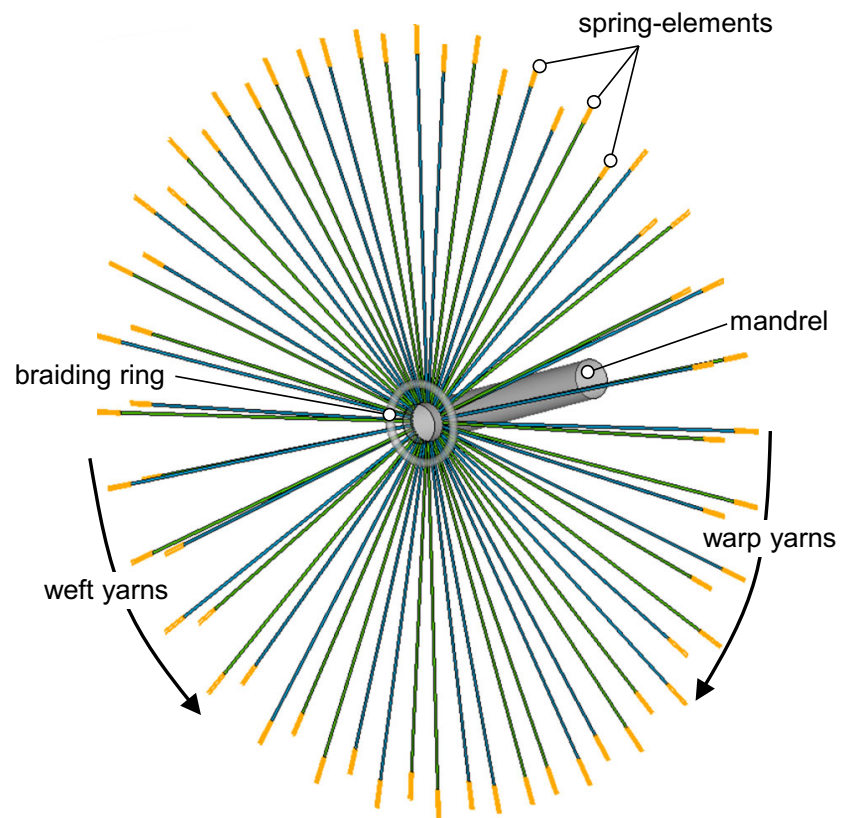

Fig. 13 Braiding process model

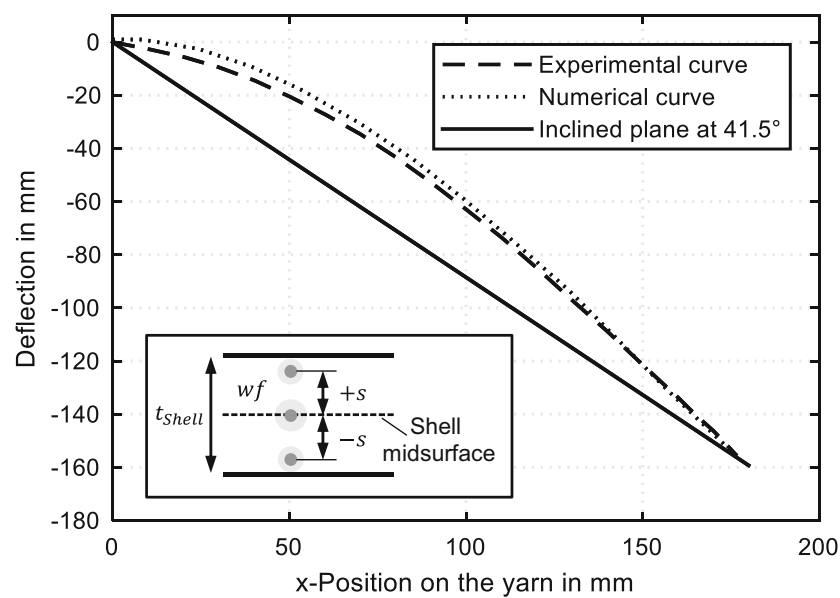

Fig. 14 Calibrated bending behavior for a yarn and the simulation yarn model

behavior. In order to model the measured bending behavior in the numerical model a user-defined integration rule through the element thickness is used. As one integration point would lead to a constant bending and tensile stiffness [40], a minimum of three integration points is necessary to decouple bending and tensile stiffness. This is done by using the *Integration_Shell keyword in LS-DYNA, where the distance $s$ of the individual integration points as well as their weighting $w f$ (see Fig. 14) are variables to be calibrated so that the model bending stiffness compares to test results. The theory of the presented method can be explained with the bending theory according to Euler-Bernoulli or Timoshenko. The latter, as an extension of the Euler-Bernoulli theory, additionally considers the shear resistance under bending load. In the work of [41] it has been shown that the shear influence is negligible for the bending behavior of a single yarn. Therefore, the following explanation is based on the Euler-Bernoulli theory. For a uniformly distributed load $q_{0}$ acting on a cantilever with a free edge and length $l$, the deflection $w(x)$ can be expressed for small deformations as

$w(x)=\frac{q_{0} l^{4}}{E I} \cdot\left(6 \frac{x^{2}}{l^{2}}-4 \frac{x^{3}}{l^{3}}+\frac{x^{4}}{l^{4}}\right)$,

where $E I$ describes the bending rigidity of the cantilever with $E$ being the Young's modulus and $I$ the second moment of inertia [42]. As the Young's modulus remains constant, the

Table 5 Values for userdefined integration rule

\begin{tabular}{lll}
\hline Integration point & $s$ & $w f$ \\
\hline 1 & 0.0 & 0.9676 \\
2 & 0.2358 & 0.0162 \\
3 & -0.2358 & 0.0162 \\
\hline
\end{tabular}


bending rigidity can only be influenced by the second moment of inertia $I$, which can be done by changing the lever arm distance $s$ or weighting $w f$ of the integration points. From [41] it is also observed, that the yarn cross-sectional shape changes during deflection which is indicated by flattening of the yarn. Although this effect is increased by smaller bending radii, the authors from [41] show that the flattening has a negligible influence on the bending rigidity, which is why the bending stiffness is assumed to be constant throughout the whole process simulation.

The parameter calibration has been performed automatically as an optimisation problem using LS-Opt. The results of this optimization are listed in Table 5, whereby 1 indicates the integration point at the shell mid-plane and 2, 3 are integration points in the $+s$ and $-s$ directions respectively, relative to the mid-plane. Figure 14 compares the deformation of the experimental yarn and the calibrated numerical model for the cantilever bending test. In both cases the dashed and dotted lines represent the deformed yarn as it extends from a cantilever to strike an inclined surface that is at $41.5^{\circ}$ to the horizontal, where a good agreement is obtained between simulation and test results.

\section{Validation of the process simulation}

An intermediate view of the shell model process simulations is shown in Fig. 15, where a properly formed biaxial braid with an average braid angle of $\pm 45^{\circ}$ has been achieved.

Numerical simulations to predict preform quality were carried out using the same machine settings as listed in Table 1. For the evaluation of the braid angle, a section of $100 \mathrm{~mm}$ length was selected over the region where the braid is properly formed, from which the variation in braid angle can be accurately measured. This is done by referring the elements 1direction (which is equal to the yarns longitudinal direction) to the mandrels longitudinal axis. These results are plotted in Fig. 16 together with test measurements, where a good agreement between the two sets of results is found. These results

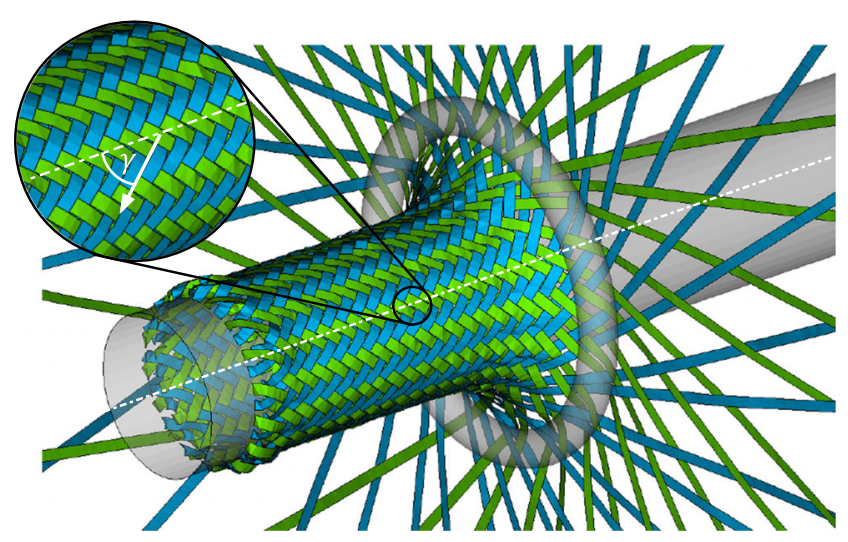

Fig. 15 Final deformed shell mesh of the braiding process simulation

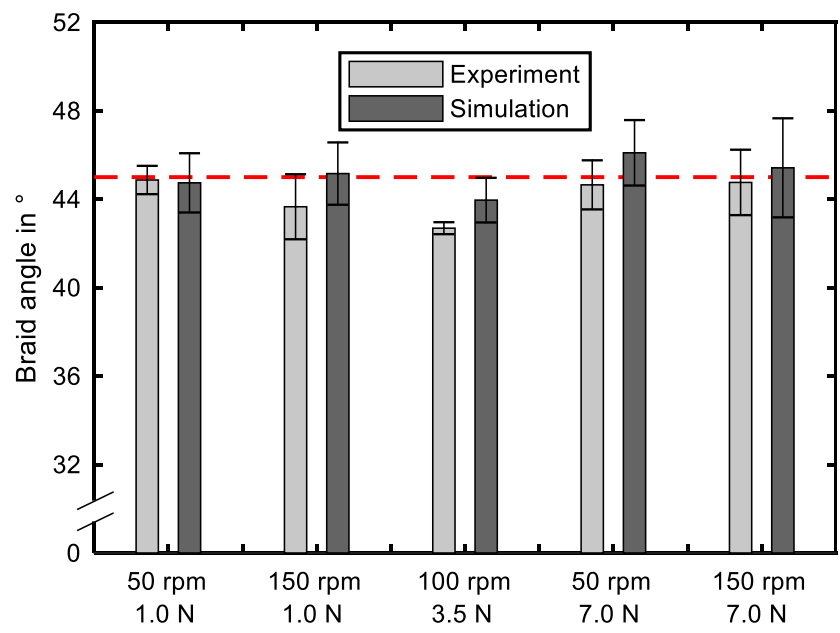

Fig. 16 Comparison of numerical and experimental braiding angle

also show that the influence of different machine settings can be captured in the numerical models, even though the simulation does slightly overestimate the braid angle, except for the machine configuration with the lowest yarn tension and process speed $(50 \mathrm{rpm} \& 1.0 \mathrm{~N})$. It is also noted that these results show similar trends for scatter of the test and simulation results. Many of these variations in the simulations occur due to the dynamic nature of the explicit analysis combined with effects like stick-slip due to friction. This can be observed, for instance, from the separation distances between yarns as they move over the braiding ring in Fig. 15.

As discussed in "Preform characterization" section fiber damage is caused by excessive friction due to interaction of the yarns during manufacture. This is most strongly influenced by the yarn tension forces and to a lesser extent by the processing speed. Simulation of the braiding process is indirectly capable of identifying potential fiber damage by examining the dissipated frictional energy in the inter-yarn contact. Figure 17 shows results of frictional energy as a function of

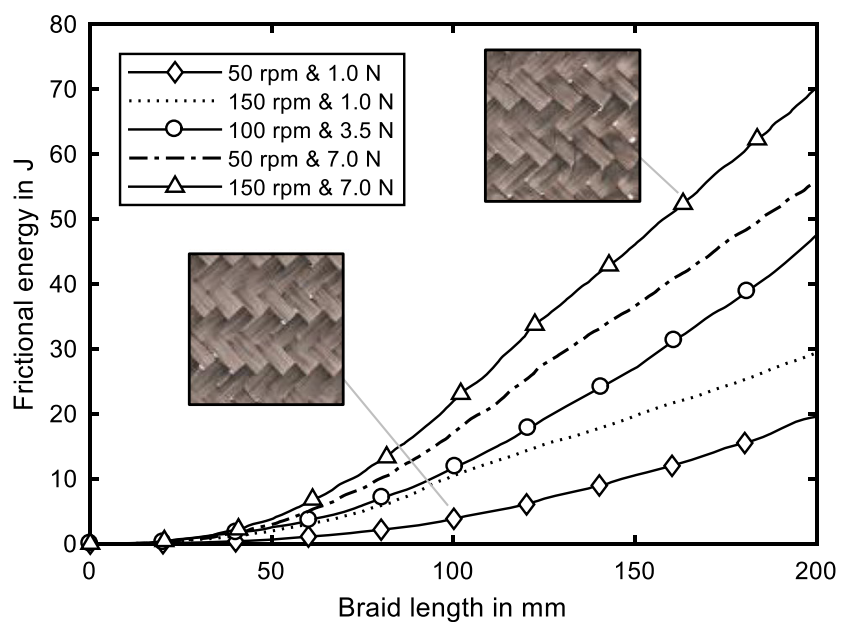

Fig. 17 Frictional energy of different machine settings in consideration of fiber damage 
formed braid length for the braid process simulation model using the different machine settings defined in Table 1. These results clearly show that larger yarn tension and faster process speeds (LS-DYNA uses a velocity dependent Coulomb friction model [43]) both increase contact friction energy, which are known experimentally to cause greater fiber damage (see Fig. 9a). Presently, this information can only provide a useful indicator of fiber damage; a larger study will be needed to try and quantify a link between friction energy and fiber damage.

\section{Textile 3D representation}

For accurate stress or infusion modelling of the textile a geometrically correct 3D solid model is essential, that should incorporate process variability. Consequently, a method is described to convert shell process simulation results to a $3 \mathrm{D}$ solid representation of the architecture. This $3 \mathrm{D}$ models can include defects, such as variability in braid angle and yarn section dimensions, and will approximate the architecture after compaction in the RTM tooling. Important information such as the material assignment and the control of the forming process are discussed and a final validation is done by comparing the resulting yarn architecture with an experiment.

\section{Model pre-processing and thermal expansion}

Performing a braiding simulation using solid elements is not feasible today, and therefore the approach adopted here is to use shells for the braiding simulation and convert these to solids for the textile model. For this conversion the preprocessor ANSA v18.1.1 from BETA CAE Systems is used. In a first step the region of interest is identified and two splines per yarn are created running through nodes along the yarns longitudinal direction (Fig. 18). An ellipse cross-section is then defined that is oriented perpendicular to the mean of the normal vector of the first two shell elements. The dimensions of the cross-section is slightly smaller than the final yarn

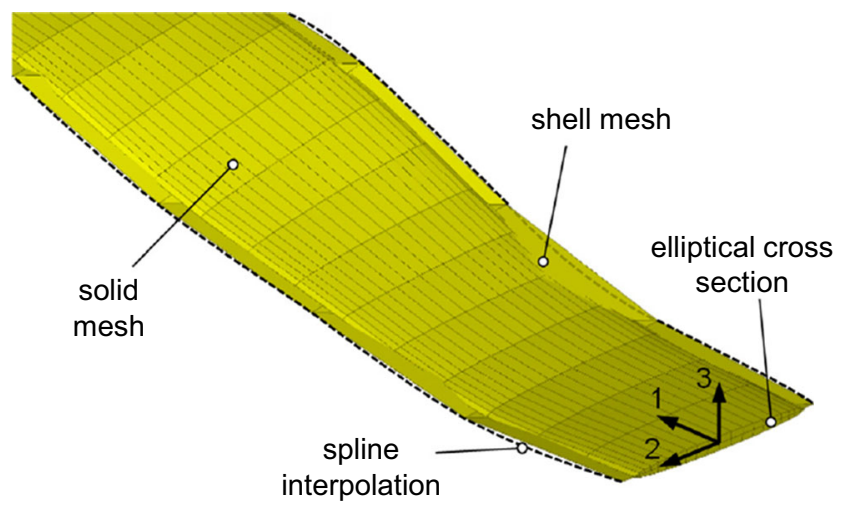

Fig. 18 Generation of the solid structure based on the results of the braiding process simulation geometry to avoid initial penetrations of solid elements between different yarns as the elliptical cross-section is extruded along the two splines.

The final step of the pre-processing is correct material assignment, as this is crucial to control the thermal expansion process that will modify yarn solid elements to the true physical yarn dimensions. This expansion can include effects of yarn dimensions variability. In [44] the authors used a 1D centerline inside the solid elements to assign the material orientation of a plain weave. This will only be valid if the yarn path can be described by means of trigonometric functions, but it will fail when dealing with a more complex architecture, since the $1 \mathrm{D}$ centerline does not provide sufficient information for a defined arrangement in space. Consequently, this work uses two splines for the solid generation process. The elliptical cross-section is cut along its major axis and meshed with an even number of elements through the thickness, since this enables the generated midsurface to be used for assignment of the 3 -direction. In combination with the edge along the longitudinal 1-direction, the material orientation can be fully defined (Fig. 18). Finally, the 'old' braid shell elements are deleted.

The initial element size of the solids is chosen to discretize the yarns width with 20 elements and the thickness with 2 elements. The element length in the yarns 1-direction is set to $1.25 \mathrm{~mm}$, which is assumed to be sufficiently small, however, no convergence study has been performed yet. For a biaxial braid with 64 yarns and a cylindrical mandrel with $85 \mathrm{~mm}$ diameter and $100 \mathrm{~mm}$ length, a total of about 275.000 elements per layer are needed for the discretization.

Initially, the solid elements have smaller dimensions than the shell elements and there is no inter-penetration of the interlaced yarns. The yarns are then expanded to correct dimension by applying artificial thermal expansion coefficients. In order to account for variations in yarn dimensions the normal distribution of these coefficients is equal to the normal distribution of the yarns cross-section. The thermal expansion is controlled by the temperature difference and the coefficient of expansion in the corresponding direction $i$, whereas the final thermal strain $\varepsilon_{i i}^{n+1}$ for the time increment $n+1$ can be calculated for a three dimensional solid element using Eq. (5) [43].

$\varepsilon_{i i}^{n+1}=\varepsilon_{i i}^{n}+\alpha_{i} \cdot\left(T^{n+1}-T^{n}\right)$

The temperature difference is set constant by default, so the final thermal strain is only controlled by the thermal expansion coefficients $\alpha_{i}$ for the principal directions 1, 2, 3 (see Fig. 18). A Poisson's ratio of $\nu=0$ is assumed during this expansion step to avoid any interaction of the principal material directions. Even though this value may not be physically correct, for this procedure it is valid and ensures an exclusive thermal strain in the desired directions without effecting the other directions. 
Fig. 19 Comparison of the final compacted textile architecture for $50 \mathrm{rpm} \& 1.0 \mathrm{~N}$; above test, below simulation

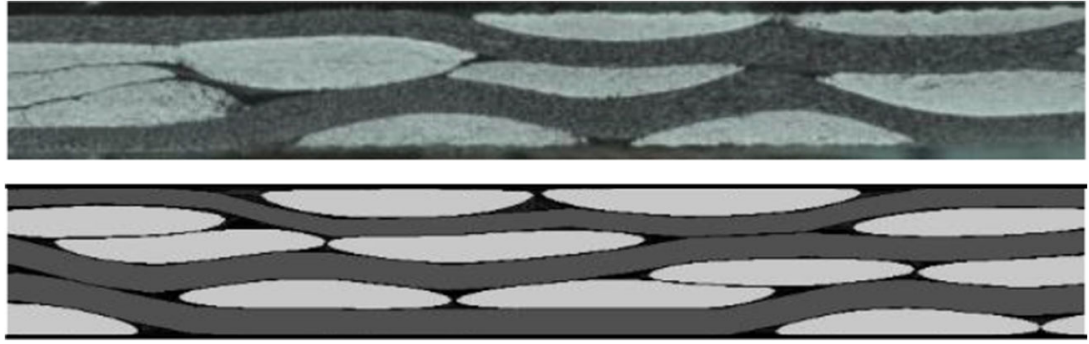

\section{Textile compaction}

After the thermal expansion has given the yarns their final geometry, the braid is then compacted in a last step. To achieve a realistic internal deformation behavior of the textile, a similar modelling approach to that presented in [25] is used, where an elasto-plastic material behavior with an artificial low yield strength is assigned to the yarns. The Poisson's ratio is set to a value near $\nu=0.5$ which ensures that volume conservation is achieved.

In order to compare experiment and simulation the example from Fig. 15 is selected which was manufactured with machine settings of $50 \mathrm{rpm}$ and $1.0 \mathrm{~N}$. After the physical manufacture of the braid, it was cut from the cylindrical mandrel and flattened on a table for measurements and infiltration. Microsections were produced from the cured plates which are shown in Fig. 19. The simulation model is produced in the same manner in which the shell braid model is separated from the mandrel and the flattening is simulated using boundary conditions, so that there is no change to the braid angle. The method presented in "Model pre-processing and thermal expansion" section is then applied to the shell mesh and the generated solids are compacted between two rigid plates, whereby the lower plate was fixed and the upper plate moved downwards with a constant displacement. The contact again uses global general contact between all parts (*Contact_Automatic_Single_Surface in LSDYNA) to avoid penetrations. A comparison of the final textile architectures is shown in Fig. 19, where it can be seen that the textile deformation is well captured by the simulation.

For a closer comparison of the test and simulation textile models the final yarn cross-sections were measured and compared with results from "Evaluation of the preform quality"

Table 6 Experimental and numerical variability of yarn cross-section $(50 \mathrm{rpm} \& 1.0 \mathrm{~N})$

\begin{tabular}{|c|c|c|c|c|c|c|}
\hline & \multicolumn{3}{|c|}{ Experiments } & \multicolumn{3}{|c|}{ Simulation } \\
\hline & $\mu[\mathrm{mm}]$ & $\sigma[\mathrm{mm}]$ & $C V[\%]$ & $\mu[\mathrm{mm}]$ & $\sigma[\mathrm{mm}]$ & $C V[\%]$ \\
\hline Yarn width & 5.80 & 0.46 & 7.87 & 5.81 & 0.44 & 7.57 \\
\hline Yarn height & 0.361 & 0.048 & 13.18 & 0.381 & 0.048 & 12.60 \\
\hline
\end{tabular}

section. These are presented in Table 6 . The comparison of the values shows that the presented method is able to accurately model variabilities with respect to the yarn geometry.

\section{Conclusions}

Radial braiding is a relative fast manufacturing process to produce near net-shape textiles. Due to the dynamic nature of the process, the preform will have variations in braid angle, yarn width, cover factor and fiber damage that are not uniformly distributed throughout the textile. For any numerical modeling of the textile, for example for permeability or mechanical properties, it is important that these variations are taken into account. This paper presents experimental investigations that have been carried out to determine the influence that different machine settings have on the final textile quality.

The experimental studies have shown that two processing properties that do influence quality of the braid architecture are machine speeds and yarn tension. Generally, these studies have shown that high processing speeds lead to oscillations in the system that increase variations in braid angles. Larger yarn tension forces appear to improve some qualities of the braid, but do also increase fiber damage. The presented studies have shown that a value of $3.5 \mathrm{~N}$ leads to a high preform quality due to lower scatter of the yarn architecture but is still low enough to result in an increase of fiber damage. In general a low process speed is recommended for carbon fibers, as it will decrease variations in the fiber architecture as well as fiber damage.

Techniques have been presented to include measured experimental variabilities within numerical models of the braid. It is shown that variations in braid angles can be obtained from explicit finite element process simulations for different machine settings and that these variations compare well to manufacturing examples. Also, the scatter of angle variations are well captured, even though the simulation slightly overestimates the experimental values for some machine parameters. Furthermore, the process simulation has been shown capable to identify possible fiber damage depending on machine settings via the level of contact friction energy. The results of the process simulation are transferred to a forming simulation, which uses a 
thermal expansion method in combination with a compaction of the textile to achieve a realistic internal fabric architecture. Variations of yarn cross-section observed in experiments are taken into account by applying variations in thermal expansion coefficients. Comparison of test microsections and numerical models do show a good agreement for the compressed textile architecture. These models of the formed textile architecture would provide an improved basis for permeability modelling with a CFD code, or for structural analysis for stiffness and strength which is the aim of ongoing research. Also, the approach used is not restricted to the textile RVE size and it is feasible to create far large models that could be analyzed at the component level.

Acknowledgements The present work is funded by the Deutsche Forschungsgemeinschaft (DFG, German Research Foundation) Project number: 323019910 , which is gratefully acknowledged by the authors. The authors acknowledge support by the state of BadenWurttemberg through bwHPC. We also thank Alexander Rack (X-ray Imaging Group - ID19, European Synchrotron Radiation Facility ESRF Grenoble) and Dascha Dobrovolskij (University of Applied Sciences, Darmstadt, Germany) for the synchrotron- $\mu \mathrm{CT}$-scan data and reconstruction, where beamtime was financed by the German Federal Ministry of Education and Research (BMBF) under grant 05M13RCA.

Funding information Open Access funding provided by Projekt DEAL.

\section{Compliance with ethical standards}

Conflict of interest The authors declare that they have no conflict of interest.

Open Access This article is licensed under a Creative Commons Attribution 4.0 International License, which permits use, sharing, adaptation, distribution and reproduction in any medium or format, as long as you give appropriate credit to the original author(s) and the source, provide a link to the Creative Commons licence, and indicate if changes were made. The images or other third party material in this article are included in the article's Creative Commons licence, unless indicated otherwise in a credit line to the material. If material is not included in the article's Creative Commons licence and your intended use is not permitted by statutory regulation or exceeds the permitted use, you will need to obtain permission directly from the copyright holder. To view a copy of this licence, visit http://creativecommons.org/licenses/by/4.0/.

\section{References}

1. Pickett A, Erber A, von Reden T, Drechsler K (2009) Comparison of analytical and finite element simulation of $2 \mathrm{D}$ braiding. Plast Rubber Compos 38(9/10):387-395

2. Tabatabaei SA (2016) Meso-finite element Modelling of textile composites using mesh superposition method. Dissertation, KU Leuven

3. Pickett A, Sirtautas J, Erber A (2009) Braiding simulation and prediction of mechanical properties. Appl Compos Mater 16(6): 345-364
4. Heieck F (2019) Potenzial einer optischen 3D-Preformanalyse zur Qualitätsbestimmung von Faser-Kunststoff-Verbunden. Dissertation, University of Stuttgart

5. Heieck F, Hermann F, Middendorf P and Schladitz K (2016) Influence of the cover factor of 2D biaxial and triaxial braided carbon composites on their in-plane mechanical properties. Composite Structures 163:114-122

6. Kyosev Y (2015) Braiding technology for textiles. Woodhead, Cambridge

7. Ebel C (2013) Effects of fiber damage on the efficiency of the braiding process, in Composites Week @ Leuven and TexComp11 Conference, Leuven

8. Matveev MY (2015) Effects of variabilities on mechanical properties of textile composites. Dissertation, The University of Nottingham

9. Rawal A, Potluri P, Steele C (2005) Geometrical modelling of the yarn paths in three-dimensional braided structures. J Ind Text 35 : 115-135

10. Rawal A, Potluri P, Steele C (2007) Prediction of yarn paths in braided structures formed on a square pyramid. J Ind Text 36: 221-226

11. van Ravenhorst JH (2018) Design tools for circular overbraiding of complex mandrels. PhD Thesis, University of Twente

12. van Ravenhorst J, Akkerman R (2016) Overbraiding simulation. In: Advances in braiding technology. Woodhead Publishing by Elsevier, Amsterdam, Boston, Cambridge, pp 431-455

13. van Ravenhorst J, Akkerman R (2016) A yarn interaction model for circular braiding. Compos A: Appl Sci Manuf 81:254-263

14. Lengersdorf M, Multhoff J, Linke M, Gries T (2013) Simulative design of overbraided pressure vessel for hydrogen storage, In: Hoa, Suong Van; Hubert, Pascal (Eds.): ICCM19 : 19th International Conference on Composite Materials, Montreal, Canada

15. Swery EE, Hans T, Bultez M, Wijaya W, Kelly P, Hinterhölzl R and Bickerton S (2017) Complete simulation process chain for the manufacturing of braided composite parts. Composites Part A 102:378-390

16. Hans T, Cichosz J, Brand M, Hinterhölzl R (2015) Finite element simulation of the braiding process for arbitrary mandrel shapes. Compos A: Appl Sci Manuf:124-132

17. Sun X, Kawashita LF, Wollmann T, Spitzer S, Langkamp A, Gude M (2018) Experimental and numerical studies on the braiding of carbon fibres over structured end-fittings for the design and manufacture of high performance hybrid shafts. Prod Eng 12:215-228

18. Grave G et al. (2009) Simulation of 3D overbraiding - solutions and challenges, Greenville, SC, USA

19. Boehler P (2019) Einzelfadenbasierte Modellierung von textilen Preform-Prozessen. Dissertation, University of Stuttgart

20. Page J, Wang J (2002) Prediction of shear force using 3D non-linear FEM analyses for a plain weave carbon fabric in a bias extension state. Finite Elem Anal Des:755-764

21. Gasser A, Boisse P, Hanklar S (1999) Mechanical behaviour of dry fabric reinforcements. 3D simulations versus biaxial tests. Comput Mater Sci 17:7-20

22. Potluri P, Sagar TV (2008) Compaction modelling of textile preforms for composite structures. Compos Struct 86(1-3):177-185

23. Green SD, Long AC, El Said BS, Hallett SR (2013) Numerical modelling of $3 \mathrm{D}$ woven preform deformations. Compos Struct 108:747-756

24. Thompson AJ, El Said B, Ivanov D, Belnoue JP-H, Hallett SR (2018) High fidelity modelling of the compression behaviour of 2D woven fabrics. Int J Solids Struct 154:104-113

25. Vinot M, Holzapfel M, Jemmali R (2015) "Numerical investigation of carbon braided composites at the Mesoscale: using computer tomography as a validation tool," in 10th European LS-DYNA Conference, Würzburg, Germany 
26. Verpoest I, Lomov SV (2005) Virtual textile composites software WiseTex: integration with micro-mechanical, permeability and structural analysis. Compos Sci Technol 65(15-16):2563-2574

27. Long AC, Brown LP (2011) Modelling the geometry of textile reinforcements for composites: TexGen," Woodhead Publishing Series in Composites Science and Engineering, pp. 239-264

28. Tabatabaei SA, Lomov SV, Verpoest I (2014) Assessment of embedded elements technique in meso-FE modelling of fibre reinforced composites. Compos Struct 107:436-446

29. De Carvalho NV, Pinho ST, Robinson P (2012) Numerical modelling of woven composites: biaxial loading. Compos A: Appl Sci Manuf 43(8):1326-1337

30. Doitrand A, Fagiano C, Irisarri F-X, Hirsekorn M (2015) Comparison between voxel and consistent meso-scale models of woven composites. Compos A: Appl Sci Manuf 73:143-154

31. Grail G, Hirsekorn M, Wendling A, Hivet G, Hambli R (2013) Consistent finite element mesh generation for meso-scale modelling of textile composites with preformed and compacted reinforcements. Compos A: Appl Sci Manuf 55:143-151

32. Bauer C, Glatt E, Wiegmann A (2017) The prediction of mechanical properties of composites and porous materials based on microCT-scans and 3D material models, in NAFEMS World Congress, Stockholm, Sweden

33. Liu Y, Straumit I, Vasiukov D, Lomov SV, Panier S (2016) "Multiscale material model for 3D composite using micro CT images geometry reconstruction," in 17th European Conference on Composite Materials (ECCM), Munich, Germany

34. Toho Tenax (2010) Datasheet of yarn material HTS40. https:// www.swiss-composite.ch/pdf/t-Tenax-Datenblatt.pdf. Accessed 14 June 2019

35. Cherif C (2011) Textile Werkstoffe für den Leichtbau - Techniken. Springer-Verlag, Verfahren, Materialien, Eigenschaften, Berlin, Heidelberg
36. Siebertz K, van Bebber D, Hochkirchen T (2010) Statistische Versuchsplanung - Design of Experiments (DoE). Springer, Berlin

37. Miene A, Herrmann AS, Göttinger M (2008) Quality assurance by digital image analysis for the preforming and draping process of dry carbon Fiber material, in SAMPE Europe International Conference and Forum, Paris, France

38. Miene A, Heumüller M, Weiland F, Weimer C (2011) Quality assurance system for aircraft structural profile preforms, in SETEC 11., Leiden, Netherlands

39. Cichosz JA (2016) Experimental characterization and numerical modeling of the mechanical response for biaxial braided composites. Dissertation, Technische Universität München

40. Nasdala L (2015) FEM-Formelsammlung Statik und Dynamik. Springer Vieweg, Wiesbaden

41. Cornelissen B, Akkerman R 2009 Analysis of yarn bending behaviour, in 17th International Conference on Composite Materials (ICCM 17), Edinburgh, United Kingdom

42. Gere J, Timoshenko S (1997) Mechanics of materials, 4th edition. PWS Publishing Company, Boston

43. Livermore Software Technology Corporation (2019) LS-DYNA theory manual. https://www.lstc.com/download/manuals. Accessed 20 March 2019

44. Nilakantan G, Cox B, Sudre O (2017) Generation of realistic stochastic virtual microstructures using a novel thermal growth method for woven fabrics and textile composites, in American Society for Composites 32nd Technical Conference, West Lafayette

Publisher's note Springer Nature remains neutral with regard to jurisdictional claims in published maps and institutional affiliations. 